\title{
A study of accretion discs around rapidly rotating neutron stars in general relativity and its applications to four low mass $\mathrm{X}$-ray binaries
}

\author{
Sudip Bhattacharyya*
}

\author{
Joint Astronomy Program, Indian Institute of Science, Bangalore 560012, India \\ Indian Institute of Astrophysics, Bangalore 560034, India \\ sudip@iiap.ernet.in
}

Received 9 July 2001 / Accepted 28 November 2001

\begin{abstract}
We calculate the accretion disc temperature profiles, disc luminosities and boundary layer luminosities for rapidly rotating neutron stars considering the full effect of general relativity. We compare the theoretical values of these quantities with their values inferred from EXOSAT data for four low mass X-ray binary sources: XB 182030, GX 17+2, GX 9+1 and GX 349+2 and constrain the values of several properties of these sources. According to our calculations, the neutron stars in GX $9+1$ and GX $349+2$ are rapidly rotating and stiffer equations of state are unfavoured.
\end{abstract}

Key words. accretion, accretion discs - relativity - stars: neutron - stars: rotation - X-rays: binaries

\section{Introduction}

A low mass $\mathrm{X}$-ray binary (LMXB) is believed to contain either a weakly magnetised neutron star or a black hole as the central accretor. The X-ray emission arises from the innermost region of the accretion disc around the compact star. In the case of a neutron star, there is an additional $\mathrm{X}$-ray component coming from the boundary layer of the star. Mitsuda et al. (1984) showed that the spectrum of a luminous LMXB can be fitted by the sum of a single temperature blackbody spectrum (believed to come from the boundary layer) and a multicolour blackbody spectrum (may originate from the accretion disc). However these authors used Newtonian models to fit the observed spectra. Near the surface of a neutron star, the accretion flow is expected to be governed by the laws of general relativity due to the presence of strong gravity. Therefore general relativistic models should be used to get the correct best-fit values of the parameters. Besides, the principal motivation behind the study of the spectral and temporal behaviours of neutron star LMXBs is to understand the properties of very high $\left(\sim 10^{15} \mathrm{~g} \mathrm{~cm}^{-3}\right)$ density matter at the neutron star core (van der Klis 2000). This is a fundamental problem of physics, which cannot be addressed by any kind of laboratory experiment. The only way to answer this question is to assume an equation of state (EOS) model for the neutron star core, to calculate

\footnotetext{
* e-mail: sudip@physics.iisc.ernet.in
}

the structure parameters of the neutron star and hence to calculate an appropriate spectral model. By fitting such models (for different chosen EOSs) to the observed data, one can hope to constrain the existing EOS models and hence to understand the properties of high density matter. However, general relativistic calculation is essential to calculate the structure parameters of a neutron star and therefore to constrain the EOS models.

It is expected that the neutron stars in LMXBs are rapidly rotating due to accretion-induced angular momentum transfer. LMXBs are thought to be the progenitors of milli-second $(\mathrm{ms})$ radio pulsars (Bhattacharya \& van den Heuvel 1991) like PSR $1937+21$ with $P \sim 1.56 \mathrm{~ms}$ (Backer et al. 1982). The recent discovery of ms $(P \sim$ $2.49 \mathrm{~ms}$ ) X-ray pulsations in XTE J1808-369 (Wijnands \& van der Klis 1998) has strengthened this hypothesis. Therefore it is necessary to calculate the structure of a rotating neutron star considering the full effect of general relativity. This was done by Cook et al. (1994) and the same procedure was used by Thampan \& Datta (1998), to calculate the luminosities of the disc and the boundary layer.

The disc temperature profile for a rapidly rotating neutron star was first calculated by Bhattacharyya et al. (2000). These authors also compared their theoretical results with the EXOSAT data (analysed by White et al. 1988) to constrain different properties of the LMXB source Cygnus X-2. The present work is a continuation of theirs, 
in which we constrain several properties of four LMXB sources: XB 1820-30, GX 17+2, GX 9+1 and GX 349+2, using the same procedure. These sources were also observed by EXOSAT and the data were analysed by White et al. (1988).

$\mathrm{XB} 1820-30$ is an atoll source which shows type I X-ray bursts. GX $17+2$ and GX $349+2$ are Z sources, of which the former shows X-ray bursts. GX $9+1$ is an atoll source. As all of them are LMXBs (van Paradijs 1995), the magnetic field of the neutron stars are believed to be decayed to lower values $\left(\sim 10^{8} \mathrm{G}\right.$; see Bhattacharya \& Datta 1996 and Bhattacharya \& van den Heuvel 1991). Therefore, we ignore the effect of the magnetic field on the accretion disc structure in our calculations.

In Sect. 2, we give the formalism of the work. We present the results and discussion in Sect. 3 and give a summary in Sect. 4.

\section{Formalism}

\subsection{Theoretical formulae}

In order to calculate the disc temperature profile, the disc luminosity and the boundary layer luminosity for a rapidly rotating neutron star considering the full effect of general relativity, we need to compute the structure of the rotating star. To do this, following Cook et al. (1994), we choose a stationary, axisymmetric, asymptotically flat and reflection-symmetric (about the equatorial plane) metric, given by

$$
\begin{aligned}
\mathrm{d} S^{2}= & g_{\mu \nu} \mathrm{d} x^{\mu} \mathrm{d} x^{\nu}(\mu, \nu=0,1,2,3) \\
= & -\mathrm{e}^{\gamma+\rho} \mathrm{d} t^{2}+\mathrm{e}^{2 \alpha}\left(\mathrm{d} \bar{r}^{2}+\bar{r}^{2} \mathrm{~d} \theta^{2}\right) \\
& +\mathrm{e}^{\gamma-\rho} \bar{r}^{2} \sin ^{2} \theta(\mathrm{d} \phi-\omega \mathrm{d} t)^{2},
\end{aligned}
$$

where the metric coefficients $\gamma, \rho, \alpha$ and the angular speed $(\omega)$ of zero-angular-momentum-observer (ZAMO) with respect to infinity, are all functions of the quasi-isotropic radial coordinate $(\bar{r})$ and polar angle $(\theta)$. The quantity $\bar{r}$ is related to the Schwarzschild-like radial coordinate $(r)$ by the equation $r=\bar{r} \mathrm{e}^{(\gamma-\rho) / 2}$. We use the geometric units $c=G=1$ in this paper.

With the assumption that the star is rigidly rotating and a perfect fluid, we solve Einstein's field equations and the equation of hydrostatic equilibrium (the equations are given in the appendix) self-consistently and numerically from the centre of the star upto infinity to obtain the metric coefficients and $\Omega_{*}$ (angular speed of neutron star with respect to infinity) as functions of $\bar{r}$ and $\theta$. The inputs of this calculation are a chosen EOS and assumed values of the central density and the ratio of polar to equatorial radii. The outputs are bulk structure parameters, such as gravitational mass $(M)$, equatorial radius $(R)$, angular momentum $(J)$, moment of inertia $(I)$ etc. of the neutron star. We can also calculate the specific disc luminosity $\left(E_{\mathrm{D}}\right)$, the specific boundary layer luminosity $\left(E_{\mathrm{BL}}\right)$, the radius $\left(r_{\mathrm{orb}}\right)$ of the innermost stable circular orbit (ISCO) and specific energy $(\tilde{E})$, specific angular momentum $(\tilde{l})$
\& angular speed $\left(\Omega_{\mathrm{K}}\right)$ of a test particle in a Keplerian orbit (see Thampan \& Datta 1998 for a description of the method of calculation).

Then we calculate the effective temperature of a geometrically thin blackbody disc, which is given by

$T_{\text {eff }}(r)=(F(r) / \sigma)^{1 / 4}$

where $\sigma$ is the Stefan-Boltzmann constant and $F$ is the $\mathrm{X}$-ray energy flux (due to viscous dissipation) per unit surface area. We calculate $F$ using the expression of Page \& Thorne (1974):

$F(r)=\frac{\dot{M}}{4 \pi r} q(r)$

where

$q(r)=-\Omega_{\mathrm{K}, r}\left(\tilde{E}-\Omega_{\mathrm{K}} \tilde{l}\right)^{-2} \int_{r_{\mathrm{in}}}^{r}\left(\tilde{E}-\Omega_{\mathrm{K}} \tilde{l}\right) \tilde{l}_{, r} \mathrm{~d} r$.

Here $r_{\text {in }}$ is the disc inner edge radius and a comma followed by a variable as subscript to a quantity represents a derivative of the quantity with respect to the variable. The values for $\tilde{E}$ and $\tilde{l}$ are given by the two conditions (circularity and extremum) for orbits (see Thampan \& Datta 1998; Bhattacharyya et al. 2000):

$$
\begin{aligned}
\tilde{E}-\omega \tilde{l} & =\frac{\mathrm{e}^{(\gamma+\rho) / 2}}{\sqrt{1-v^{2}}} \\
\tilde{l} & =\frac{v \bar{r} \mathrm{e}^{(\gamma-\rho) / 2}}{\sqrt{1-v^{2}}}
\end{aligned}
$$

where $v=(\Omega-\omega) \bar{r} \mathrm{e}^{-\rho} \sin \theta$ is the physical velocity of the matter. The equation of motion in the azimuthal direction and that in the time direction yield the Keplerian angular speed as

$\Omega_{\mathrm{K}}=\mathrm{e}^{2 \rho(\bar{r})} \frac{\tilde{l} / \bar{r}^{2}}{(\tilde{E}-\omega \tilde{l})}+\omega(\bar{r})$

Equation (4), that is exactly valid for a black hole accretor, is also valid for a neutron star accretor, if the disc does not touch the star. But, for a disc that extends up to the surface of the neutron star, the torque at the disc inner edge will not vanish, and Eq. (4) will not be strictly valid for such a case. However, for a very rapidly rotating neutron star, the angular speed of a particle at the disc inner edge will be close to the spin rate of the star, and hence the torque is expected to be negligible. According to Bhattacharyya et al. (2000), even when the spin rate is not large, Eq. (4) should give approximately correct results. This is because, Page \& Thorne (1974) argued that the error in the calculation of $T_{\text {eff }}$ will not be substantial outside a radial distance $1.1 r_{\text {in }}$. In our calculation, we find that almost whole of the disc X-ray flux comes from welloutside this radial distance.

Equation (2) gives the effective disc temperature $T_{\text {eff }}(r)$ with respect to an observer comoving with the disc. For our purpose, this expression of the temperature must be changed to that in the observer's frame, taking into account the gravitational redshift and the rotational Doppler 
effect. In order to keep our analysis tractable, we use the expression given in Hanawa (1989) for this modification:

$1+z=\left(1-\frac{3 M}{r}\right)^{-1 / 2}$

With this correction for $(1+z)$, we define a temperature relevant for observations $\left(T_{\mathrm{obs}}^{\max }\right)$ as:

$T_{\mathrm{obs}}^{\max }=\frac{1}{1+z} T_{\mathrm{eff}}^{\max }$

where the superscript "max" denotes the maximum temperature value in the profile. $T_{\mathrm{obs}}^{\max }$ is related to the colour temperature $\left(T_{\text {col }}^{\max }\right)$ by $T_{\text {obs }}^{\max }=T_{\text {col }}^{\max } / f$, where $f$ is the colour factor (Shimura \& Takahara 1995; see also Bhattacharyya et al. 2000 for details). We compare the observationally inferred value of the maximum temperature with $T_{\text {col }}^{\max }$. It is to be remembered that Eq. (8) is valid for a non-rotating neutron star and a "face-on" (i.e., inclination angle $i=0$ ) observation. However, the error involved by these assumptions is expected to be well within the error bars considered in Sect. 2.3.

The structure of a neutron star for a given EOS is described uniquely by two parameters: the gravitational mass $(M)$ and the angular speed $\left(\Omega_{*}\right)$. For each adopted EOS, we construct constant $M$ equilibrium sequences with $\Omega_{*}$ varying from the non-rotating case (static limit) up to the centrifugal mass shed limit (rotation rate at which inwardly directed gravitational forces are balanced by outwardly directed centrifugal forces). So we are able to calculate $T_{\mathrm{eff}}^{\max }, E_{\mathrm{D}}$ and $E_{\mathrm{BL}}$ as functions of $M$ and $\Omega_{*}$ for a chosen EOS model.

\subsection{Equations of state}

The neutron star structure parameters are quite sensitive to the chosen EOS models. For the purpose of a general study, we have used the same four EOSs as considered in Bhattacharyya et al. (2000), namely, (A) Pandharipande (1971), (B) Baldo et al. (1997), (C) Walecka (1974) and (D) Sahu et al. (1993). EOS model (A) is for hyperonic matter. It is assumed that hyperonic potentials are similar to the nucleon-nucleon potentials, but altered suitably to represent the different isospin states. Model (B) is a microscopic EOS for asymmetric nuclear matter, derived from the Brueckner-Bethe-Goldstone many-body theory with explicit three-body terms. The three-body force parameters are adjusted to give a reasonable saturation point for nuclear matter. EOS model (C) corresponds to pure neutron matter and is based on a mean-field theory with exchange of scaler and (isoscalar) vector mesons representing the nuclear interaction. Model (D) is a field theoretical EOS for neutron-rich matter in beta equilibrium based on the chiral sigma model. The model includes an isoscalar vector field generated dynamically and reproduces the empirical values of the nuclear matter saturation density and binding energy and also the isospin symmetry coefficient for asymmetric nuclear matter. Of these, model (A) is soft,
Table 1. Best-fit values of the parameters (see text) for four LMXBs.

\begin{tabular}{|c|c|c|c|c|}
\hline \multirow[t]{2}{*}{ Source name } & \multirow[t]{2}{*}{$\begin{array}{c}T_{\mathrm{col}}^{\max } \\
\left(10^{7} \mathrm{~K}\right)\end{array}$} & \multicolumn{2}{|r|}{ 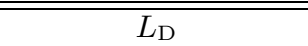 } & \multirow[t]{2}{*}{$\begin{array}{c}L_{\mathrm{BL}} \\
\left(10^{38} \mathrm{ergs} \mathrm{s}^{-1}\right)\end{array}$} \\
\hline & & $\cos i=0.2$ & $2 \cos i=0.8$ & \\
\hline XB 1820-30 & 1.59 & 1.49 & 0.37 & 0.26 \\
\hline GX $17+2$ & 1.76 & 6.49 & 1.62 & 0.71 \\
\hline GX 9+1 & 2.25 & 6.01 & 1.50 & 0.25 \\
\hline GX $349+2$ & 2.07 & 8.54 & 2.14 & 0.48 \\
\hline
\end{tabular}

(B) is intermediate in stiffness and (C) \& (D) are stiff EOSs, with (D) as the stiffest.

\subsection{Constraining procedure}

We choose four LMXB sources observed by EXOSAT (data analysed by White et al. 1988). For each source, we take the best-fit values of the parameters $T_{\text {col }}^{\max }, L_{\mathrm{D}}$ and $L_{\mathrm{BL}}$, where $L$ denotes the luminosity. On the other hand, for the chosen values of $f$, the accretion rate $(\dot{M})$ and $M$, we theoretically calculate the values of $T_{\mathrm{col}}^{\max }$, $L_{\mathrm{D}}$ and $L_{\mathrm{BL}}$ as functions of $\Omega_{*}$, for an assumed EOS model. Then comparing these theoretical values with the observed ones, we constrain different parameters of the chosen source (see Bhattacharyya et al. 2000 for detailed description). However, to take into account the uncertainties in the fitting procedure and in the value of $z$, and also those arising due to the simplicity of the model, we consider a range of acceptable values for $T_{\mathrm{col}}^{\max }, L_{\mathrm{D}}$ and $L_{\mathrm{BL}}$. We take two combinations of deviations around the bestfit values, namely, $(10 \%, 25 \%)$ and $(20 \%, 50 \%)$, where the first number in parentheses corresponds to the error in $T_{\mathrm{col}}^{\max }$ and the second to the error in the best-fit luminosities.

\section{Results and discussion}

In this paper, we calculate gravitational mass sequences for different EOS models and constrain several properties of four LMXB sources. For the neutron star in each of the sources, we assume $M=1.4 M_{\odot}$ (i.e., the canonical mass value for neutron stars). We take two values for $\cos i$ ( $i$ is the inclination angle of the source) for each source, namely, 0.2 and 0.8 . These two widely different values ensure the sufficient generality of our results. For the four sources, the best-fit values (White et al. 1988) of the parameters $T_{\mathrm{col}}^{\max }, L_{\mathrm{D}}$ and $L_{\mathrm{BL}}$ are given in Table 1.

We take the distance $(D)$ of the source XB 1820-30 as $6.4 \mathrm{kpc}$ (Bloser et al. 2000). We assume $D=8 \mathrm{kpc}$ for both GX $17+2$ and GX $9+1$, as their locations are believed to be near the galactic centre (Deutsch et al. 1999; Hertz et al. 1990) and distance of the galactic centre is $7.9 \pm 0.3 \mathrm{kpc}$, as concluded by McNamara et al. (2000). For GX $349+2$, we take $D=9 \mathrm{kpc}$ (Deutsch et al. 1999).

We display the constrained values with the help of four tables. It is to be noted that here $\dot{M}$ is presented in unit of $\dot{M}_{\mathrm{e}}=1.4 \times 10^{17} \mathrm{M} / M_{\odot} \mathrm{g} \mathrm{s}^{-1}$. The Eddington rate is 
$\dot{M}_{\mathrm{e}} / \eta$, with $\eta=E_{\mathrm{BL}}+E_{\mathrm{D}}$. Therefore, as the actual value of $\eta$ is much lesser than 1.0 (generally not greater than 0.3 and for rapidly rotating neutron star, typically less than 0.2 ), the value of Eddington accretion rate is much higher than $\dot{M}_{\mathrm{e}}$. For all the sources, as the stiffness of the EOS models increases, the absolute values of the allowed spin frequencies $\left(\nu_{*}\right)$ and rotational frequencies in the ISCO $\left(\nu_{\text {in }}\right)$ decreases. This is because, for a stiffer EOS model, neutron star radius is higher and it can support lesser amount of rotation. The energy conversion efficiency is also lesser for a stiffer EOS model (as the neutron star for this case is less compact) and therefore higher accretion rate is needed to generate the observed luminosity (as seen from the tables). In the following, we describe the results for four sources in four subsections and give a general discussion in the last subsection.

\subsection{XB 1820-30}

We display the allowed ranges of different parameters for the source XB 1820-30 in Table 2. It is seen that for $\cos i=0.2$, the spin frequency $\left(\nu_{*}\right)$ of the neutron star comes out to be very high. But in the case of $\cos i=0.8$, it is not possible to constrain $\nu_{*}$ for $(20 \%, 50 \%)$ uncertainty set (for all EOS models) and for both the uncertainty sets (for EOS model D). The ranges of the colour factor are in general consistent with the results of Shimura \& Takahara $(f \sim 1.7-2.0)$ and Borozdin et al. (1999) $(f=2.6)$. However, some discrepancy can be noted with the latter one for softer EOS models $\& \cos i=0.2$. The value of the rotational frequency in the ISCO $\left(\nu_{\text {in }}\right)$ comes out to be $\sim 1 \mathrm{kHz}$ for all the cases. The values of the stellar equatorial radius are in the range $8-21 \mathrm{~km}$. The peak of the disk effective temperature occurs in the radial range $18-30 \mathrm{~km}$, and always well-outside (by several kilometers) the neutron star's surface. This shows the validity of Eq. (4), as discussed in Sect. 2. The overall range of the accretion rate comes out to be $0.5-31.4 \dot{M}_{\mathrm{e}}$.

\section{2. $G X 17+2$}

Table 3 shows the results for the source GX $17+2$. Here the ranges of $\nu_{*}$ are similar to those for XB 1820-30. But for GX $17+2$, the value of $i$ is expected to be moderately high (Titarchuk et al. 2001) and therefore $\cos i$ is not possibly as high as 0.8 . It is, therefore, quite likely that the neutron star in this source is rapidly rotating, although no decisive statement can be made. The allowed values for $f$ for GX $17+2$ is systematically lower than those for XB 182030 , and in the case of softer EOS models \& $\cos i=0.2$ they do not tally with the result of Borozdin et al. (1999). The allowed values of $\nu_{\text {in }}$ coms out to be $\sim 1 \mathrm{kHz}$, but for EOS model (A) \& $\cos i=0.8,2 \mathrm{kHz}$ value is also possible. The ranges of $R$ and $r_{\mathrm{eff}}^{\max }$ are similar to those for $\mathrm{XB} 1820-30$ and the allowed values of the accretion rate are in the range $2.0-131.0 \dot{M}_{\mathrm{e}}$.

\section{3. $G \times 9+1$}

The results for the source GX $9+1$ are given in Table 4 . For this source, $\nu_{*}$-value comes out to be very high for all the EOS models and for both the $\cos i$-values. Here, the allowed values for $f$ are inconsistent with Borozdin et al. (1999) for softer EOS models \& $\cos i=0.2$. The allowed values of $\nu_{\text {in }}$ are $\sim 1 \mathrm{kHz}$ and the allowed ranges of $R$ and $r_{\text {eff }}^{\max }$ are $10-21 \mathrm{~km}$ and $18-30 \mathrm{~km}$ respectively. The allowed values of accretion rate for this source come out to be in the range $3.8-116.7 \dot{M}_{\mathrm{e}}$.

\section{4. $G \times 349+2$}

The allowed ranges of different parameters for the source GX 349+2 are given in Table 5. As is the case for GX 9+1, here also the value of $\nu_{*}$ comes out to be very high for all the chosen EOS models and $\cos i$-values. The allowed values for $f$ in general tally with the results of Shimura \& Takahara (1995), but like other three sources do not match with the result of Borozdin et al. (1999) for softer EOS models $\& \cos i=0.2$. Here $\nu_{\text {in }}$ comes out to be $\sim 1 \mathrm{kHz}$, and $R \& r_{\mathrm{eff}}^{\max }$ are in the ranges $10-21 \mathrm{~km}$ and $18-30 \mathrm{~km}$ respectively (like GX $9+1$ ). The accretion rate for this source is in the range $4.5-168.5 \dot{M}_{\mathrm{e}}$.

\subsection{General discussion}

Here we have constrained the values of several properties of four LMXB sources. For all of them, the accretion rates come out to be very high (always $\geq 0.5 \dot{M}_{\mathrm{e}}$ ). This is in accord with the fact that these are very luminous sources.

The rotation rate of neutron star in each of the sources is very high (close to the mass shed limit) for $\cos i=0.2$. This is because, the values of $L_{\mathrm{BL}} / L_{\mathrm{D}}$ are very low for these cases (see Thampan \& Datta 1998; Bhattacharyya et al. 2000). But, for $\cos i=0.8$, rotation rate cannot be constrained effectively for the sources XB 1820-30 and GX $17+2$. Therefore, for these two sources, no general conclusion (about the values of $\Omega_{*}$ ) can be drawn. However, the allowed ranges (combined for all the cases considered in a table) of $\Omega_{*} / \Omega_{*, \mathrm{~ms}}$ are $0.93-1.00$ and $0.75-1.00$ for the other two sources GX $9+1$ and GX $349+2$ respectively (here $\Omega_{* \text {,ms }}$ is the $\Omega_{*}$ at the mass shed limit; see Bhattacharyya et al. 2000 for the mass shed limit values). Therefore the neutron stars in these two sources can be concluded to be rapidly rotating in general.

Our calculated allowed ranges for $f$ are in accord with the results obtained by Shimura \& Takahara (1995). However, if we take the value $f=2.6$ (obtained by Borozdin et al. 1999), one would require a very stiff EOS model or a mass greater than $M=1.4 M_{\odot}$ for most of the cases with $\cos i=0.2$.

High frequency quasi-periodic-oscillations ( $\mathrm{kHz}$ QPO) have been observed for three (XB 1820-30, GX 17+2 and GX $349+2)$ of the chosen sources. The observed maximum $\mathrm{kHz}$ QPO frequencies are $1.100 \mathrm{kHz}$ (XB 1820$30), 1.080 \mathrm{kHz}(\mathrm{GX} \mathrm{17+2)}$ and $1.020 \mathrm{kHz}$ (GX 349+2) 
Table 2. Observational constraints for various EOS models: (A), (B), (C), (D) for the source XB 1820-30. L and U stand for lower and upper limits. The parameters are $f$ (colour factor), $\nu_{*}$ (rotational frequency of the neutron star), $\nu_{\text {in }}$ (rotational frequency in the ISCO), $R$ (equatorial radius of the neutron star), $r_{\mathrm{eff}}^{\max }$ (radius where the effective temperature of the disc is maximum) and $\dot{M}$ (the accretion rate). The limits are for $25 \%$ uncertainty in luminosities and $10 \%$ uncertainty in the colour temperature. Values in brackets are for $50 \%$ uncertainty in luminosities and $20 \%$ uncertainty in the colour temperature. $i$ is the inclination angle of the source with respect to the observer. The mass of the neutron star is assumed to be $1.4 M_{\odot}$. The accretion rate is given in unit of $\dot{M}_{\mathrm{e}}=1.4 \times 10^{17} M / M_{\odot} \mathrm{g} \mathrm{s}^{-1}$, where $M$ is the neutron star mass.

\begin{tabular}{|c|c|c|c|c|c|c|c|c|}
\hline EOS & $\cos i$ & & $f$ & $\begin{array}{l}\nu_{*} \\
\mathrm{kHz}\end{array}$ & $\begin{array}{l}\nu_{\text {in }} \\
\mathrm{kHz}\end{array}$ & $\begin{array}{l}R \\
\mathrm{~km}\end{array}$ & $\begin{array}{l}r_{\text {eff }}^{\max } \\
\mathrm{km}\end{array}$ & $\begin{array}{l}\dot{M} \\
\dot{M}_{\mathrm{e}}\end{array}$ \\
\hline \multirow[t]{2}{*}{$(\mathrm{A})$} & 0.2 & $\mathrm{~L}$ & $1.31[1.10]$ & $1.751[1.726]$ & $1.756[1.756]$ & $11.2[10.2]$ & $18.6[18.1]$ & $\overline{7.7[3.8]}$ \\
\hline & & $\mathrm{U}$ & $1.91[2.41]$ & $1.755[1.755]$ & $1.819[2.078]$ & $11.4[11.4]$ & $18.7[18.7]$ & $16.1[20.3]$ \\
\hline \multirow[t]{2}{*}{ (B) } & 0.2 & $\mathrm{~L}$ & $1.45[1.30]$ & $1.103[1.059]$ & $1.137[1.126]$ & $15.0[13.7]$ & $23.0[21.6]$ & $9.7[4.5]$ \\
\hline & & $\mathrm{U}$ & $2.07[2.61]$ & $1.112[1.113]$ & $1.197[1.372]$ & $15.5[15.6]$ & $23.5[23.6]$ & $19.4[24.4]$ \\
\hline \multirow[t]{2}{*}{ (C) } & 0.2 & $\mathrm{~L}$ & $1.49[1.30]$ & $0.961[0.913]$ & $0.979[0.973]$ & $16.5[15.0]$ & $24.8[23.1]$ & $10.4[4.9]$ \\
\hline & & $\mathrm{U}$ & $2.12[2.71]$ & $0.968[0.968]$ & $1.042[1.206]$ & $17.2[17.2]$ & $25.5[25.6]$ & $21.2[26.7]$ \\
\hline \multirow[t]{2}{*}{ (D) } & 0.2 & $\mathrm{~L}$ & $1.59[1.40]$ & $0.735[0.687]$ & $0.748[0.743]$ & $19.9[17.7]$ & $29.1[26.5]$ & $12.2[5.6]$ \\
\hline & & $\mathrm{U}$ & $2.25[2.81]$ & $0.740[0.740]$ & $0.795[0.941]$ & $20.6[20.7]$ & $30.0[30.1]$ & $25.0[31.4]$ \\
\hline \multirow[t]{2}{*}{$(\mathrm{A})$} & 0.8 & $\mathrm{~L}$ & $1.79[1.50]$ & $1.463[0.000]$ & $1.822[1.571]$ & $9.9[7.5]$ & $18.1[18.1]$ & $1.2[0.5]$ \\
\hline & & $\mathrm{U}$ & $3.06[4.70]$ & $1.751[1.754]$ & $2.165[2.165]$ & 11.2[11.4] & $20.4[22.3]$ & $4.5[6.4]$ \\
\hline \multirow[t]{2}{*}{ (B) } & 0.8 & $\mathrm{~L}$ & $1.94[1.71]$ & $0.498[0.000]$ & $1.207[1.152]$ & $11.3[11.0]$ & $20.2[20.2]$ & $1.4[0.9]$ \\
\hline & & $\mathrm{U}$ & $3.22[4.20]$ & $1.102[1.110]$ & $1.782[1.782]$ & $14.9[15.4]$ & $22.9[23.4]$ & $5.6[7.9]$ \\
\hline \multirow[t]{2}{*}{ (C) } & 0.8 & $\mathrm{~L}$ & $1.99[1.70]$ & $0.175[0.000]$ & $1.046[0.991]$ & $12.3[12.3]$ & $21.5[21.0]$ & $1.5[1.0]$ \\
\hline & & $\mathrm{U}$ & $3.36[4.02]$ & $0.960[0.966]$ & $1.573[1.573]$ & $16.5[17.0]$ & $24.7[25.4]$ & $6.1[8.5]$ \\
\hline \multirow[t]{2}{*}{ (D) } & 0.8 & $\mathrm{~L}$ & $2.11[1.80]$ & $0.000[0.000]$ & $0.806[0.758]$ & $14.7[14.7]$ & $23.1[23.1]$ & $1.8[1.2]$ \\
\hline & & $\mathrm{U}$ & $3.30[3.90]$ & $0.733[0.739]$ & $1.212[1.212]$ & $19.7[20.5]$ & $28.9[29.8]$ & $7.2[9.9]$ \\
\hline
\end{tabular}

Table 3. Observational constraints for various EOS models: (A), (B), (C), (D) for the source GX 17+2. Other specifications are same as in Table 2.

\begin{tabular}{|c|c|c|c|c|c|c|c|c|}
\hline \multirow[t]{2}{*}{$\overline{\mathrm{EOS}}$} & \multirow[t]{2}{*}{$\cos i$} & & \multirow[t]{2}{*}{$\overline{\overline{f f}}$} & \multirow{2}{*}{$\begin{array}{l}\nu_{*} \\
\mathrm{kHz}\end{array}$} & \multirow{2}{*}{$\begin{array}{l}\nu_{\text {in }} \\
\mathrm{kHz}\end{array}$} & \multirow{2}{*}{$\begin{array}{l}R \\
\mathrm{~km}\end{array}$} & \multirow{2}{*}{$\begin{array}{l}r_{\text {eff }}^{\max } \\
\mathrm{km}\end{array}$} & \multirow{2}{*}{$\begin{array}{l}\dot{M} \\
\dot{M}_{\mathrm{e}}\end{array}$} \\
\hline & & & & & & & & \\
\hline \multirow[t]{2}{*}{ (A) } & 0.2 & $\mathrm{~L}$ & $1.01[1.00]$ & $1.754[1.748]$ & $1.756[1.756]$ & $11.4[11.0]$ & $18.7[18.5]$ & $36.1[19.8]$ \\
\hline & & $\mathrm{U}$ & $1.43[1.82]$ & $1.755[1.755]$ & $1.773[1.869]$ & $11.4[11.4]$ & $18.7[18.7]$ & $68.7[82.6]$ \\
\hline \multirow[t]{2}{*}{ (B) } & 0.2 & $\mathrm{~L}$ & $1.12[1.00]$ & $1.108[1.097]$ & $1.128[1.122]$ & $15.3[14.7]$ & $23.3[22.6]$ & $44.4[24.4]$ \\
\hline & & $\mathrm{U}$ & $1.57[1.96]$ & $1.113[1.113]$ & $1.163[1.236]$ & $15.6[15.7]$ & $23.6[23.6]$ & $82.6[101.7]$ \\
\hline \multirow[t]{2}{*}{ (C) } & 0.2 & $\mathrm{~L}$ & $1.15[1.00]$ & $0.966[0.954]$ & $0.974[0.971]$ & $16.9[16.0]$ & $25.2[24.2]$ & $47.5[26.1]$ \\
\hline & & $\mathrm{U}$ & $1.62[2.01]$ & $0.968[0.968]$ & $1.000[1.091]$ & $17.2[17.2]$ & $25.5[25.6]$ & $88.5[111.5]$ \\
\hline \multirow[t]{2}{*}{ (D) } & 0.2 & $\bar{L}$ & $1.22[1.03]$ & $0.738[0.728]$ & $0.744[0.742]$ & $20.3[19.2]$ & $29.7[28.3]$ & $55.9[30.7]$ \\
\hline & & $\mathrm{U}$ & $1.72[2.13]$ & $0.740[0.740]$ & $0.766[0.849]$ & $20.7[20.7]$ & $30.1[30.1]$ & $106.4[131.0]$ \\
\hline \multirow[t]{2}{*}{$(\mathrm{A})$} & 0.8 & $\mathrm{~L}$ & $1.39[1.20]$ & $1.702[0.000]$ & $1.782[1.571]$ & $9.9[7.5]$ & $18.1[18.1]$ & $6.6[2.0]$ \\
\hline & & $\mathrm{U}$ & $2.20[3.72]$ & $1.754[1.755]$ & $2.166[2.166]$ & $11.3[11.4]$ & $18.6[22.3]$ & $18.5[25.5]$ \\
\hline \multirow[t]{2}{*}{ (B) } & 0.8 & $\mathrm{~L}$ & $1.53[1.31]$ & $1.009[0.000]$ & $1.172[1.141]$ & $13.1[11.0]$ & $21.1[20.2]$ & $7.7[3.2]$ \\
\hline & & $\mathrm{U}$ & $2.31[3.35]$ & $1.107[1.111]$ & $1.463[1.782]$ & $15.2[15.5]$ & $23.2[23.5]$ & $22.8[30.7]$ \\
\hline \multirow[t]{2}{*}{ (C) } & 0.8 & $\bar{L}$ & $1.61[1.30]$ & $0.858[0.000]$ & $1.010[0.983]$ & $14.3[12.3]$ & $22.4[21.0]$ & $8.3[3.6]$ \\
\hline & & $\mathrm{U}$ & $2.33[3.20]$ & $0.965[0.967]$ & $1.289[1.568]$ & $16.8[17.1]$ & $25.1[25.4]$ & $25.0[32.9]$ \\
\hline \multirow[t]{2}{*}{ (D) } & 0.8 & $\mathrm{~L}$ & $1.66[1.41]$ & $0.631[0.000]$ & $0.775[0.750]$ & $16.8[14.7]$ & $25.4[23.1]$ & $9.5[4.4]$ \\
\hline & & $\mathrm{U}$ & $2.47[3.10]$ & $0.737[0.740]$ & $1.011[1.212]$ & $20.2[20.6]$ & $29.5[30.0]$ & $29.3[38.6]$ \\
\hline
\end{tabular}

(van der Klis 2000). Now, as pointed out in Bhattacharyya et al. (2000), the maximum possible frequency (i.e., the shortest time scale) of the system should be given by the rotational frequency in ISCO $\left(\nu_{\text {in }}\right.$; Col. 5 of the tables). Therefore, the stiffest EOS model D is unfavoured for $\cos i=0.2$ for the source XB 1820-30, as the maximum value of $\nu_{\text {in }}(=0.941 \mathrm{kHz}$, Table 2$)$ is less than the observed maximum $\mathrm{kHz}$ QPO frequency. For the same reason, EOS model D is unfavoured for $\cos i=0.2$ for the source GX $17+2$. It can also be seen from Table 3 that if we use only the narrower limits on the luminosities and colour temperature, EOS model D (for $\cos i=0.8$ ) and 
Table 4. Observational constraints for various EOS models: (A), (B), (C), (D) for the source GX 9+1. Other specifications are same as in Table 2.

\begin{tabular}{|c|c|c|c|c|c|c|c|c|}
\hline \multirow[t]{2}{*}{ EOS } & \multirow[t]{2}{*}{$\overline{c o s} i$} & & \multirow[t]{2}{*}{$\bar{f}$} & \multirow{2}{*}{$\begin{array}{l}\nu_{*} \\
\mathrm{kHz}\end{array}$} & \multirow{2}{*}{$\begin{array}{l}\nu_{\mathrm{in}} \\
\mathrm{kHz}\end{array}$} & \multirow{2}{*}{$\begin{array}{l}R \\
\mathrm{~km}\end{array}$} & \multirow{2}{*}{$\begin{array}{l}r_{\mathrm{eff}}^{\max } \\
\mathrm{km}\end{array}$} & \multirow{2}{*}{$\begin{array}{l}\dot{M} \\
\dot{M}_{\mathrm{e}}\end{array}$} \\
\hline & & & & & & & & \\
\hline \multirow[t]{2}{*}{ (A) } & 0.2 & $\bar{L}$ & $1.33[1.13]$ & $1.755[1.755]$ & $1.756[1.756]$ & $11.4[11.4]$ & $18.7[18.7]$ & $36.9[22.8]$ \\
\hline & & $\mathrm{U}$ & $1.85[2.25]$ & $1.755[1.755]$ & $1.756[1.761]$ & $11.4[11.4]$ & $18.7[18.7]$ & $59.9[72.0]$ \\
\hline \multirow[t]{2}{*}{ (B) } & 0.2 & $\bar{L}$ & $1.47[1.24]$ & $1.112[1.110]$ & $1.120[1.117]$ & $15.6[15.4]$ & $23.6[23.4]$ & $\overline{43.4[27.4]}$ \\
\hline & & $\mathrm{U}$ & $2.04[2.49]$ & $1.114[1.114]$ & $1.130[1.147]$ & $15.7[15.7]$ & $23.6[23.7]$ & $73.6[90.6]$ \\
\hline \multirow[t]{2}{*}{ (C) } & 0.2 & $\mathrm{~L}$ & $1.51[1.28]$ & $0.968[0.967]$ & $0.970[0.970]$ & $17.2[17.1]$ & $25.5[25.4]$ & $47.5[29.3]$ \\
\hline & & $\mathrm{U}$ & $2.09[2.56]$ & $0.968[0.968]$ & $0.975[0.986]$ & $17.3[17.3]$ & $25.6[25.6]$ & $80.7[99.3]$ \\
\hline \multirow[t]{2}{*}{ (D) } & 0.2 & $\mathrm{~L}$ & $1.61[1.36]$ & $0.740[0.739]$ & $0.742[0.741]$ & $20.7[20.5]$ & $30.1[29.9]$ & $55.9[34.4]$ \\
\hline & & $\mathrm{U}$ & $2.24[2.74]$ & $0.740[0.740]$ & $0.745[0.754]$ & $20.7[20.7]$ & $30.1[30.1]$ & $94.9[116.7]$ \\
\hline \multirow[t]{2}{*}{ (A) } & 0.8 & $\mathrm{~L}$ & $1.84[1.61]$ & $1.752[1.728]$ & $1.756[1.756]$ & $11.2[10.3]$ & $18.6[18.1]$ & $7.9[3.8]$ \\
\hline & & $\mathrm{U}$ & $2.69[3.52]$ & $1.755[1.755]$ & $1.815[2.064]$ & $11.4[11.4]$ & $18.7[18.7]$ & $16.5[20.3]$ \\
\hline \multirow[t]{2}{*}{ (B) } & 0.8 & $\mathrm{~L}$ & $2.05[1.80]$ & $1.103[1.064]$ & $1.136[1.126]$ & $15.0[13.8]$ & $22.9[21.7]$ & $9.7[4.6]$ \\
\hline & & $\mathrm{U}$ & $2.92[3.72]$ & $1.112[1.113]$ & $1.200[1.361]$ & $15.5[15.6]$ & $23.5[23.6]$ & $19.4[24.4]$ \\
\hline \multirow[t]{2}{*}{ (C) } & 0.8 & $\mathrm{~L}$ & $2.10[1.80]$ & $0.961[0.919]$ & $0.978[0.972]$ & $16.5[15.0]$ & $24.8[23.2]$ & $10.4[5.0]$ \\
\hline & & $\mathrm{U}$ & $3.00[3.81]$ & $0.968[0.968]$ & $1.041[1.195]$ & $17.2[17.2]$ & $25.5[25.6]$ & $21.2[26.7]$ \\
\hline \multirow[t]{2}{*}{ (D) } & 0.8 & $\bar{L}$ & $2.24[1.92]$ & $0.734[0.692]$ & $0.748[0.743]$ & $19.8[17.8]$ & $29.0[26.7]$ & $12.2[5.7]$ \\
\hline & & $\mathrm{U}$ & $3.19[4.00]$ & $0.740[0.740]$ & $0.802[0.932]$ & $20.6[20.7]$ & $30.0[30.1]$ & $25.0[31.4]$ \\
\hline
\end{tabular}

Table 5. Observational constraints for various EOS models: (A), (B), (C), (D) for the source GX 349+2. Other specifications are same as in Table 2 .

\begin{tabular}{|c|c|c|c|c|c|c|c|c|}
\hline \multirow[t]{2}{*}{$\overline{\mathrm{EOS}}$} & \multirow[t]{2}{*}{$\overline{\cos i}$} & & \multirow[t]{2}{*}{$\overline{f f}$} & \multirow{2}{*}{$\begin{array}{l}\nu_{*} \\
\mathrm{kHz} \\
\end{array}$} & \multirow{2}{*}{$\begin{array}{l}\nu_{\text {in }} \\
\mathrm{kHz}\end{array}$} & \multirow{2}{*}{$\begin{array}{l}R \\
\mathrm{~km}\end{array}$} & \multirow{2}{*}{$\begin{array}{l}r_{\text {eff }}^{\max } \\
\mathrm{km}\end{array}$} & \multirow{2}{*}{$\begin{array}{l}\dot{M} \\
\dot{M}_{\mathrm{e}} \\
\end{array}$} \\
\hline & & & & & & & & \\
\hline \multirow[t]{2}{*}{$(\mathrm{A})$} & 0.2 & $\mathrm{~L}$ & $1.12[1.00]$ & $1.755[1.754]$ & $1.756[1.756]$ & $11.4[11.4]$ & $18.7[18.7]$ & $50.9[30.7]$ \\
\hline & & $\mathrm{U}$ & $1.55[1.92]$ & $1.755[1.755]$ & $1.756[1.771]$ & $11.4[11.4]$ & $18.7[18.7]$ & $86.4[103.9]$ \\
\hline \multirow[t]{2}{*}{ (B) } & 0.2 & $\mathrm{~L}$ & $1.24[1.04]$ & $1.112[1.109]$ & $1.122[1.119]$ & $15.5[15.3]$ & $23.5[23.3]$ & $61.2[37.7]$ \\
\hline & & $\mathrm{U}$ & $1.72[2.11]$ & $1.113[1.114]$ & $1.137[1.159]$ & $15.7[15.7]$ & $23.6[23.7]$ & $106.3[130.8]$ \\
\hline \multirow[t]{2}{*}{ (C) } & 0.2 & $\mathrm{~L}$ & $1.27[1.08]$ & $0.968[0.966]$ & $0.971[0.970]$ & $17.2[17.0]$ & $25.5[25.3]$ & $67.1[40.4]$ \\
\hline & & $\mathrm{U}$ & $1.76[2.17]$ & $0.968[0.968]$ & $0.979[1.000]$ & $17.2[17.3]$ & $25.6[25.6]$ & $116.6[140.1]$ \\
\hline \multirow[t]{2}{*}{ (D) } & 0.2 & $\mathrm{~L}$ & $1.35[1.14]$ & $0.740[0.738]$ & $0.742[0.741]$ & $20.6[20.4]$ & $30.0[29.7]$ & $78.8[47.5]$ \\
\hline & & $\mathrm{U}$ & $1.88[2.31]$ & $0.740[0.740]$ & $0.748[0.765]$ & $20.7[20.7]$ & $30.1[30.1]$ & $136.9[168.5]$ \\
\hline \multirow[t]{2}{*}{ (A) } & 0.8 & $\mathrm{~L}$ & $1.55[1.34]$ & $1.748[1.678]$ & $1.760[1.756]$ & $11.0[9.7]$ & $18.4[18.1]$ & $10.6[4.5]$ \\
\hline & & $\mathrm{U}$ & $2.29[3.10]$ & $1.755[1.755]$ & $1.879[2.147]$ & $11.4[11.4]$ & $18.7[18.7]$ & $23.3[30.0]$ \\
\hline \multirow[t]{2}{*}{ (B) } & 0.8 & $\mathrm{~L}$ & $1.71[1.50]$ & $1.096[0.955]$ & $1.148[1.129]$ & $14.6[12.7]$ & $22.6[20.7]$ & $13.1[5.2]$ \\
\hline & & $\mathrm{U}$ & $2.47[3.22]$ & $1.110[1.112]$ & $1.244[1.532]$ & $15.4[15.6]$ & $23.4[23.6]$ & $28.0[36.0]$ \\
\hline \multirow[t]{2}{*}{ (C) } & 0.8 & $\mathrm{~L}$ & $1.76[1.53]$ & $0.953[0.798]$ & $0.984[0.974]$ & $16.0[13.8]$ & $24.2[21.9]$ & $14.0[5.6]$ \\
\hline & & $\mathrm{U}$ & $2.54[3.30]$ & $0.967[0.968]$ & $1.093[1.350]$ & $17.1[17.2]$ & $25.4[25.5]$ & $30.7[38.6]$ \\
\hline \multirow[t]{2}{*}{ (D) } & 0.8 & $\bar{L}$ & $1.87[1.61]$ & $0.727[0.557]$ & $0.753[0.744]$ & $19.1[16.2]$ & $28.2[24.6]$ & $16.5[6.3]$ \\
\hline & & $\mathrm{U}$ & $2.69[3.40]$ & $0.739[0.740]$ & $0.845[1.069]$ & $20.6[20.7]$ & $29.9[30.1]$ & $36.0[46.4]$ \\
\hline
\end{tabular}

EOS model $\mathrm{C}$ (for $\cos i=0.2$ ) are unfavoured for the same source. Same is true for EOS model D for the source GX $349+2$. As we also see from Table 5, EOS model C is unfavoured for $\cos i=0.2$ for this source. Therefore, we may conclude that the stiffer EOS models are unfavoured by our results.

We have ignored the magnetic fields of the neutron stars in our calculations. Therefore, the necessary condition for the validity of our results is that the Alfvén radius
$\left(r_{\mathrm{A}}\right)$ be less than the radius of the inner edge of the disc. This condition will always be valid if $R>r_{\mathrm{A}}$ holds. Here $r_{\mathrm{A}}$ is given by (Shapiro \& Teukolsky 1983),

$r_{\mathrm{A}}=2.9 \times 10^{8}\left(\frac{\dot{M}}{\dot{M}_{\mathrm{e}}}\right)^{-2 / 7} \mu_{30}^{4 / 7}\left(\frac{M}{M_{\odot}}\right)^{-3 / 7}$

where $M$ is the mass of the neutron star, $\mu_{30}$ is the magnetic moment in units of $10^{30} \mathrm{G} \mathrm{cm}^{3}$ and $r_{\mathrm{A}}$ is in $\mathrm{cm}$. With typical values of the parameters for the chosen sources 
$\left(R=10 \mathrm{~km}, M=1.4 M_{\odot}\right.$ and $\left.\dot{M}=10 \dot{M}_{\mathrm{e}}\right)$, the upper limit of the neutron star surface magnetic field comes out to be about $2 \times 10^{8} \mathrm{G}$. Therefore, our results are in general valid for the neutron star magnetic field upto of the order of $10^{8} \mathrm{G}$. This is a reasonable value for the magnetic field of neutron stars in LMXBs, as mentioned in Sect. 1.

It is also to be noted that our results are valid for a thin blackbody disc. However, as the spectra of the sources were well-fitted by a multicolour blackbody (plus a blackbody, presumably coming from the boundary layer; White et al. 1988), the assumption of thin blackbody disc may be correct.

\section{Summary}

In this paper, we have constrained the values of two neutron star parameters (spin frequency and equatorial radius) for the four LMXBs: XB 1820-30, GX 17+2, GX 9+1 and GX 349+2. We have also calculated the allowed ranges of the colour factor (for accretion disc), rotational frequency $\left(\nu_{\text {in }}\right)$ of a particle in the ISCO, the radius $\left(r_{\text {eff }}^{\max }\right)$ where the effective disc temperature is maximum and the accretion rate for these sources. These have been done for a chosen mass $1.4 M_{\odot}$ (canonical mass) of the neutron stars and two values of inclination angles $(\cos i=0.2,0.8)$. The whole work has been repeated for four EOS models (from very soft to very stiff).

We have drawn the following main conclusions from our study. A comparison between the $\mathrm{kHz}$ QPO frequencies (observed from the sources) and our calculated values of $\nu_{\text {in }}$ has shown that the stiffer EOS models are unfavoured. By the constraining procedure, we have got very high accretion rates for all the sources, which is in accordance with their high luminosities. The neutron stars in the sources GX $9+1$ and GX $349+2$ have been found to be very rapidly rotating, and those in the other two sources may also be rapidly rotating (although we can not say decisively). This is in accordance with the belief that LMXBs are the progenitors of millisecond pulsars. It also shows that while calculating the spectral models, taking the rapid rotation of neutron stars into account is very important.

It is difficult to constrain EOS models effectively with the poor quality EXOSAT data. However, the present generation $\mathrm{X}$-ray satellites Chandra and $X M M$ have much better resolving power. For example, the resolving power of Chandra HETGS is $60-1000$ in the energy range $0.5-10.0 \mathrm{keV}$. The future generation $\mathrm{X}$-ray satellite Constellation- $X$ will have even better resolving power (upto 3000). With the spectral data of these X-ray observatories, it may be possible to constrain EOS models and other parameters of LMXBs effectively. Therefore we propose that it is essential to compute EOS-dependent general relativistic spectral models to utilise these good quality data in a fruitful way.
Acknowledgements. We acknowledge Arun Thampan for providing us with the neutron star structure calculation code and for discussions. We thank Dipankar Bhattacharya for reading the manuscript and giving valuable suggestions. We also thank Ranjeev Misra and late Bhaskar Datta for discussions and Pijush Bhattacharjee for encouragement.

\section{Appendix}

Here we give Einstein's field equations and the equation of hydrostatic equilibrium that were solved for the computation of the structure of the rapidly rotating neutron star. For an axisymmetric and equatorial plane symmetric configuration, the computational domain in spherical polar coordinates covers $0 \leq r \leq \infty$ and $0 \leq \theta \leq \pi / 2$. For numerical convenience, we make a change of variables $(r \rightarrow s$ and $\theta \rightarrow \mu)$ given by $\tilde{r}=\tilde{r_{\mathrm{e}}} \frac{s}{1-s}$ and $\theta=\cos ^{-1} \mu$, where $\overline{r_{\mathrm{e}}}$ is the quasi-isotropic radial coordinate of the equator. It is easy to see that $s$ and $\mu$ vary in the range $0 \leq s \leq 1$ and $0 \leq \mu \leq 1$ and at the equator $s=0.5$.

The four Einstein's equations (Cook et al. 1994) to solve are given below:

$$
\begin{aligned}
\rho(s, \mu) & =-\mathrm{e}^{-\gamma / 2} \sum_{n=0}^{\infty} P_{2 n}(\mu)\left[\left(\frac{1-s}{s}\right)^{2 n+1} \int_{0}^{s} \frac{\mathrm{d} s^{\prime} s^{2 n}}{\left(1-s^{\prime}\right)^{2 n+2}}\right. \\
& \times \int_{0}^{1} \mathrm{~d} \mu^{\prime} P_{2 n}\left(\mu^{\prime}\right) \tilde{S}_{\rho}\left(s^{\prime}, \mu^{\prime}\right)+\left(\frac{s}{1-s}\right)^{2 n} \\
& \left.\times \int_{s}^{1} \frac{\mathrm{d} s^{\prime}\left(1-s^{\prime}\right)^{2 n-1}}{s^{\prime 2 n+1}} \int_{0}^{1} \mathrm{~d} \mu^{\prime} P_{2 n}\left(\mu^{\prime}\right) \tilde{S}_{\rho}\left(s^{\prime}, \mu^{\prime}\right)\right] \\
\gamma(s, \mu) & =-\frac{2 \mathrm{e}^{-\gamma / 2}}{\pi} \sum_{n=1}^{\infty} \frac{\sin [(2 n-1) \theta]}{(2 n-1) \sin \theta}\left[\left(\frac{1-s}{s}\right)^{2 n}\right. \\
& \times \int_{0}^{s} \frac{\mathrm{d} s^{\prime} s^{\prime 2 n-1}}{\left(1-s^{\prime}\right)^{2 n+1}} \int_{0}^{1} \mathrm{~d} \mu^{\prime} \sin \left[(2 n-1) \theta^{\prime}\right] \tilde{S}_{\gamma}\left(s^{\prime}, \mu^{\prime}\right) \\
& +\left(\frac{s}{1-s}\right)^{2 n-2} \int_{s}^{1} \frac{\mathrm{d} s^{\prime}\left(1-s^{\prime}\right)^{2 n-3}}{s^{\prime 2 n-1}} \\
& \left.\times \int_{0}^{1} \mathrm{~d} \mu^{\prime} \sin \left[(2 n-1) \theta^{\prime}\right] \tilde{S}_{\gamma}\left(s^{\prime}, \mu^{\prime}\right)\right]
\end{aligned}
$$

$$
\begin{aligned}
\hat{\omega}(s, \mu) & =-\mathrm{e}^{(2 \rho-\gamma) / 2} \sum_{n=1}^{\infty} \frac{P_{2 n-1}^{1}(\mu)}{2 n(2 n-1) \sin \theta}\left[\left(\frac{1-s}{s}\right)^{2 n+1}\right. \\
& \times \int_{0}^{s} \frac{\mathrm{d} s^{\prime} s^{2 n}}{\left(1-s^{\prime}\right)^{2 n+2}} \int_{0}^{1} \mathrm{~d} \mu^{\prime} \sin \theta^{\prime} P_{2 n-1}^{1}\left(\mu^{\prime}\right) \tilde{S}_{\hat{\omega}}\left(s^{\prime}, \mu^{\prime}\right) \\
& +\left(\frac{s}{1-s}\right)^{2 n-2} \int_{s}^{1} \frac{\mathrm{d} s^{\prime}\left(1-s^{\prime}\right)^{2 n-3}}{s^{2 n-1}} \\
& \left.\times \int_{0}^{1} \mathrm{~d} \mu^{\prime} \sin \theta^{\prime} P_{2 n-1}^{1}\left(\mu^{\prime}\right) \tilde{S}_{\hat{\omega}}\left(s^{\prime}, \mu^{\prime}\right)\right]
\end{aligned}
$$




$$
\begin{aligned}
\alpha_{, \mu}= & -\frac{1}{2}\left(\rho_{, \mu}+\gamma_{, \mu}\right)-\left\{\left(1-\mu^{2}\right)\left[1+s(1-s) \gamma_{, s}\right]^{2}\right. \\
& \left.+\left[-\mu+\left(1-\mu^{2}\right) \gamma_{, \mu}\right]^{2}\right\}-1\left[\frac { 1 } { 2 } \left\{s(1-s)\left[s(1-s) \gamma_{, s}\right]_{, s}\right.\right. \\
& +s^{2}(1-s)^{2} \gamma_{, s}^{2}-\left[\left(1-\mu^{2}\right) \gamma_{, \mu}\right]_{, \mu} \\
& \left.-\gamma_{, \mu}\left[-\mu+\left(1-\mu^{2}\right) \gamma_{, \mu}\right]\right\}\left[-\mu+\left(1-\mu^{2}\right) \gamma_{, \mu}\right] \\
& +\frac{1}{4}\left[s^{2}(1-s)^{2}\left(\rho_{, s}+\gamma_{, s}\right)^{2}-\left(1-\mu^{2}\right)\left(\rho_{, \mu}+\gamma_{, \mu}\right)^{2}\right] \\
& \times\left[-\mu+\left(1-\mu^{2}\right) \gamma_{, \mu}\right]-s(1-s)\left(1-\mu^{2}\right) \\
& \times\left(\frac{1}{2}\left(\rho, \gamma_{, s}\right)\left(\rho_{, \mu}+\gamma_{, \mu}\right)+\gamma, s \mu+\gamma_{, s} \gamma_{, \mu}\right) \\
& \times\left[1+s(1-s) \gamma_{, s}\right]+s(1-s) \mu \gamma_{, s}\left[1+s(1-s) \gamma_{, s}\right] \\
& +\frac{1}{4}\left(1-\mu^{2}\right) \mathrm{e}^{-2 \rho}\left\{2 \frac{s^{3}}{1-s}\left(1-\mu^{2}\right) \hat{\omega}_{, s} \hat{\omega}_{, \mu}\right. \\
& \times\left[1+s(1-s) \gamma_{, s}\right]-\left(s^{4} \hat{\omega}_{, s}^{2}-\frac{s^{2}}{(1-s)^{2}}\left(1-\mu^{2}\right) \hat{\omega}_{, s}^{2}\right) \\
& \left.\left.\times\left[-\mu+\left(1-\mu^{2}\right) \gamma_{, \mu}\right]\right\}\right]
\end{aligned}
$$

where $P_{n}(\mu)$ are the Legendre polynomials, $P_{n}^{m}(\mu)$ are the associated Legendre polynomials and $\sin (n \theta)$ is a function of $\mu$ through $\theta=\cos ^{-1} \mu$. The effective sources $\tilde{S}$ 's are defined as (Cook et al. 1994)

$$
\begin{aligned}
& \tilde{S}_{\rho}(s, \mu)=\mathrm{e}^{\gamma / 2}\left[8 \pi \mathrm{e}^{2 \alpha} \tilde{r}_{\mathrm{e}}^{2}(\tilde{\epsilon}+\tilde{P})\left(\frac{s}{1-s}\right)^{2} \frac{1+\tilde{v}^{2}}{1-\tilde{v}^{2}}\right. \\
& +\left(\frac{s}{1-s}\right)^{2}\left(1-\mu^{2}\right) \mathrm{e}^{-2 \rho}\left\{\left[s(1-s) \hat{\omega}_{, s}\right]^{2}+\left(1-\mu^{2}\right) \hat{\omega}_{, \mu}^{2}\right\} \\
& +s(1-s) \gamma_{, s}-\mu \gamma_{, \mu}+\frac{\rho}{2}\left\{16 \pi \mathrm{e}^{2 \alpha} \tilde{r}_{\mathrm{e}}^{2} \tilde{P}\left(\frac{s}{1-s}\right)^{2}\right. \\
& -s(1-s) \gamma_{, s}\left(\frac{s(1-s)}{2} \gamma_{, s}+1\right) \\
& \left.\left.\quad-\gamma_{, \mu}\left(\frac{1-\mu^{2}}{2} \gamma_{, \mu}-\mu\right)\right\}\right]
\end{aligned}
$$

$$
\begin{aligned}
\tilde{S}_{\gamma}(s, \mu)= & \mathrm{e}^{\gamma / 2}\left[16 \pi \mathrm{e}^{2 \alpha} \tilde{r}_{\mathrm{e}}^{2} \tilde{P}\left(\frac{s}{1-s}\right)^{2}\right. \\
& +\frac{\gamma}{2}\left\{16 \pi \mathrm{e}^{2 \alpha} \tilde{r}_{\mathrm{e}}^{2} \tilde{P}\left(\frac{s}{1-s}\right)^{2}\right. \\
& \left.\left.-\frac{s^{2}(1-s)^{2}}{2} \gamma_{, s}^{2}-\frac{1-\mu^{2}}{2} \gamma_{, \mu}^{2}\right\}\right]
\end{aligned}
$$

$$
\begin{aligned}
& \tilde{S}_{\hat{\omega}}(s, \mu)=\mathrm{e}^{(\gamma-2 \rho) / 2}\left[-16 \pi \mathrm{e}^{2 \alpha} \frac{\left(\hat{\Omega}_{*}-\hat{\omega}\right)}{1-\tilde{v}^{2}} \tilde{r}_{\mathrm{e}}^{2}(\tilde{\epsilon}+\tilde{P})\right. \\
& \quad \times\left(\frac{s}{1-s}\right)^{2}+\hat{\omega}\left\{-8 \pi \mathrm{e}^{2 \alpha} \tilde{r}_{\mathrm{e}}^{2} \frac{\left(1+\tilde{v}^{2}\right) \tilde{\epsilon}+2 \tilde{v}^{2} \tilde{P}}{1-\tilde{v}^{2}}\right. \\
& \quad \times\left(\frac{s}{1-s}\right)^{2}-s(1-s)\left(2 \rho_{, s}+\frac{1}{2} \gamma_{, s}\right)+\mu\left(2 \rho_{, \mu}+\frac{1}{2} \gamma_{, \mu}\right) \\
& \quad+\frac{s^{2}(1-s)^{2}}{4}\left(4 \rho_{, s}^{2}-\gamma_{, s}^{2}\right)+\frac{1-\mu^{2}}{4}\left(4 \rho_{, \mu}^{2}-\gamma_{, \mu}^{2}\right) \\
& \left.\left.\quad-\left(1-\mu^{2}\right) e^{-2 \rho}\left(s^{4} \hat{\omega}_{, s}^{2}+\frac{s^{2}\left(1-\mu^{2}\right)}{(1-s)^{2}} \hat{\omega}_{, \mu}^{2}\right)\right\}\right]
\end{aligned}
$$

where $\hat{\omega} \equiv \tilde{r}_{\mathrm{e}} \tilde{\omega}$ and $\hat{\Omega}_{*} \equiv \tilde{r}_{\mathrm{e}} \tilde{\Omega}_{*}$. Here the tilde over a variable represents the corresponding dimensionless quantity and the variables $\epsilon$ and $P$ (not to be confused with Legendre polynomials, that always have a function of " $n$ " as the subscript) represent mass-energy density and pressure respectively.

The equation of hydrostatic equilibrium for a barytropic fluid is

$$
\begin{aligned}
h(\tilde{P})-h_{\mathrm{p}} & \equiv \int_{\tilde{P}_{\mathrm{p}}}^{\tilde{P}} \frac{\mathrm{d} \tilde{P}}{\tilde{\epsilon}+\tilde{P}} \\
& =\ln u^{\mathrm{t}}-\ln u_{\mathrm{p}}^{\mathrm{t}}-\int_{\tilde{\Omega}_{*, \mathrm{c}}}^{\tilde{\Omega}_{*}} F\left(\tilde{\Omega}_{*}\right) \mathrm{d} \tilde{\Omega}_{*}
\end{aligned}
$$

where $h(\tilde{P})$ is the dimensionless specific enthalpy as a function of pressure and $\tilde{P}_{\mathrm{p}}, u_{\mathrm{p}}^{\mathrm{t}}$ and $h_{\mathrm{p}}$ are the dimensionless values of pressure, t-component of the four-velocity and the specific enthalpy at the pole. The quantity $\tilde{\Omega}_{*, \mathrm{c}}$ is the (dimensionless) central value of the angular speed, which on the rotation axis is constant and equal to its value at the pole. The quantity $F\left(\tilde{\Omega}_{*}\right)=u^{\mathrm{t}} u_{\phi}$ is obtained from an integrability condition on the equation of hydrostatic equilibrium. Choosing the form of this function fixes the rotation law for the matter. Following Komatsu et al. (1989), we set it to $F\left(\tilde{\Omega}_{*}\right)=A^{2}\left(\tilde{\Omega}_{*, \mathrm{c}}-\tilde{\Omega}_{*}\right)$. Here $A$ is a rotation constant such that rigid rotation is achieved in the limit $A \rightarrow \infty$. An appropriately chosen value of $h_{\mathrm{p}}$ defines the surface of the star.

\section{References}

Backer, D. C., Kulkarni, S. R., Heiles, C., Davis, M. M., \& Goss, W. M. 1982, Nature, 300, 615

Baldo, M., Bombaci, I., \& Burgio, G. F. 1997, A\&A, 328, 274

Bhattacharya, D., \& Datta, B. 1996, MNRAS, 282, 1059

Bhattacharya, D., \& van den Heuvel, E. P. J. 1991, Phys. Rep., 203,1

Bhattacharyya, S., Thampan, A. V., Misra, R., \& Datta, B. 2000, ApJ, 542, 473

Bloser, P. F., Grindlay, J. E., Kaaret, P., et al. 2000, ApJ, 542, 1000

Borozdin, K., Revnivtsev, M., Trudolyubov, S., Shrader, C., \& Titarchuk, L. 1999, ApJ, 517, 367

Cook, G. B., Shapiro, S. L., \& Teukolsky, S. A. 1994, ApJ, 424, 823 
Deutsch, E. W., Margon, B., Anderson, S. F., Wachter, S., \& Goss, W. M. 1999, ApJ, 524, 406

Hanawa, T. 1989, ApJ, 341, 948

Hertz, P., Norris, J. P., Wood, K. S., Vaughan, B. A., \& Michelson, P. F. 1990, ApJ, 354, 267

Komatsu, H., Eriguchi, Y., \& Hachisu, I. 1989, MNRAS, 237, 355

McNamara, D. H., Madsen, J. B., Barnes, J., \& Ericksen, B. F. 2000, PASP, 112, 202

Mitsuda, K., Inoue, H., Koyama, K., Makishima, K., et al. 1984, PASJ, 36, 741

Page, D. N., \& Thorne, K. S. 1974, ApJ, 191, 499

Pandharipande, V. R. 1971, Nucl. Phys. A, 178, 123
Sahu, P. K., Basu, R., \& Datta, B. 1993, ApJ, 416, 267 Shapiro, S. L., \& Teukolsky, S. A. 1983, in Black Holes, White Dwarfs, and Neutron Stars (New York: John Wiley \& Sons) Shimura, T., \& Takahara, F. 1995, ApJ, 445, 780

Titarchuk, L. G., Bradshaw, C. F., Geldzahler, B. J., \& Fomalont, E. B. 2001 [astro-ph/0105559]

Thampan, A. V., \& Datta, B. 1998, MNRAS, 297, 570

van der Klis, M. 2000, ARA\&A, 38, 717

van Paradijs, J. 1995, in X-Ray Binaries, ed. W. H. G. Lewin, J. van Paradijs, \& E. P. J. van den Heuvel, 536

Walecka, J. D. 1974, Ann. Phys., 83, 491

White, N. E., Stella, L., \& Parmar, A. N. 1988, ApJ, 324, 363

Wijnands, R., \& van der Klis, M. 1998, Nature, 394, 344 